Italy, Department of Clinical Sciences and Community Health, Milano, Italy; ${ }^{4}$ AOU Meyer, Florence, Italy, Paediatrics, Firenze, Italy; ${ }^{5}$ University of Siena, Siena, Italy, Department of Medical Biotechnology, Siena, Italy; ${ }^{6}$ Pediatric Rheumatology Unit, ASST Centro Traumatologico Ortopedico G. PiniCTO, Milano, Italy, Department of Rheumatology and Medical Sciences, Milano, Italy

Background: The ankle is one of the most commonly affected sites in juvenile idiopathic arthritis (JIA). This region has a complex anatomical structure owing to the presence of multiple joint recesses and surrounding tendons. While the prognostic value of ultrasound (US)-detected arthritis has been investigated in recent studies, the role of tenosynovitis in JIA remains still unexplored.

Objectives: To investigate: 1) US features of ankle involvement in JIA at disease onset; 2) the predictive value of US-detected tenosynovitis in ankles with clinically active disease of children with new-onset JIA.

Methods: The clinical charts of all consecutive patients with new-onset JIA between May 2018 and January 2020 at study centres (Policlinico and G.Pini Hospitals of Milan) and with clinically active ankle disease among the joints affected were reviewed retrospectively. Data on ankle US assessment were retrieved and patients were then stratified as follows: 1) patients with detection on US of isolated arthritis in at least one of the joint recesses of the ankle region; 2) patients with detection on US of tenosynovitis in at least one of the tendon compartments of the ankle irrespective of the presence of concomitant arthritis. For each of these two categories, estimation of patients who were able to achieve clinical disease remission at 12 months since disease onset was evaluated.

Results: Twenty-seven new-onset JIA patients were found to have clinical involvement of the ankle among the joints affected. Nine of them (33.3\%) showed on US isolated arthritis of the ankle, whereas US-detected tenosynovitis was found in $18(66.7 \%)$ patients. The amount of patients who were able to achieve disease remission at 12 -months was the same $(66.7 \%)$ for both patients with and without US-detected tenosynovitis in the ankle (12/18 and 6/9 patients, respectively). In patients with US-detected tenosynovitis and clinical disease remission at 12 months, the lateral tendon compartment (LTC) was the tendon site more frequently affected by pathology $(75.0 \%)$. Patients with US-detected tenosynovitis that did not achieve clinical disease remission at follow-up had the highest frequency of tendon pathology on US in the medial tendon compartment (MTC) (83.3\%). The anterior tendon compartment was the less frequently affected tendon compartment of the ankle in all patients (33.3\% in both patients with and without clinical remission of disease at the 12-months follow-up visit).

Conclusion: US-detected tenosynovitis of the ankle is a common finding in patients with new-onset JIA with clinically ankle disease activity and is more frequent than the detection on US of isolated arthritis. The MTC and LTC are the tendon compartments more commonly affected on US. The detection on US of tenosynovitis at disease onset in ankles with clinical disease activity did not seem to affect the change to achieve the overall clinical disease remission compared to patients without tendon pathology but with joint disease in the ankle region.

Disclosure of Interests: None declared

DOI: 10.1136/annrheumdis-2021-eular.1931

\section{POS1308 \\ PREDICTORS OF DISEASE PROGRESSION IN A MONOCENTRIC COHORT OF 100 PATIENTS WITH CHILDHOOD-ONSET SYSTEMIC LUPUS ERYTHEMATOSUS}

C. Chighizola ${ }^{1,2}$, I. Pontikaki ${ }^{2}$, S. Costi ${ }^{1,2}$, G. Armentaro ${ }^{1,2}$, M. Gattinara ${ }^{2}$, T. Giani ${ }^{3}$, R. Cimaz ${ }^{1,2,4} .{ }^{1}$ University of Milan, Department of Clinical Sciences and Community Health, Milan, Italy; ${ }^{2}$ ASST G. Pini \& CTO, Pediatric Rheumatology Unit, Milan, Italy; ${ }^{3}$ Azienda Ospedaliero Universitaria Meyer, Rheumatology, Florence, Italy; ${ }^{4}$ University of Milan, RECAP_RD, Milan, Italy

Background: Childhood-onset systemic lupus erythematosus (CSLE) accounts for $10-20 \%$ of overall cases, usually presenting in early adolescence. SLE in children is characterized by a severe clinical course.

Objectives: This study describes the evolution of clinical manifestations of CSLE, with the aims of i) identifying biomarkers predictive of disease progression and ii) assessing the effects of treatment on disease course.

Methods: Laboratory and clinical data of CSLE patients followed in our Paediatric Rheumatology Unit were retrospectively collected at diagnosis and during follow-up. Continuous data were expressed as median (interquartile range [IQR]) and categorical data as percentages. The association between categorical variables was assessed by chi-squared test, the correlation between variables was tested by Pearson's test. Univariate linear regression analyses were performed to investigate the relationship between the rate of new disease manifestations at follow-up and candidate predictors. Statistical analysis was performed using GraphPadPrism v6. P values $<0.05$ were regarded statistically significant.

Results: One-hundred patients (89\% of female gender) with cSLE were included in this study, with a median age at disease onset of 13 years (10.515). Clinical details are presented in Table 1. Complement levels were reduced in 71 patients (C3 in 62 and C4 in 65). At a median follow-up of 118 months (57-239), the disease progressed in 93 patients, with a median of two new manifestations per patient (1-3). No correlation emerged between the number of new disease manifestations and both age at diagnosis and disease duration. Among serological biomarkers, a reduction in complement fractions at diagnosis emerged as the only predictor of new clinical manifestations due to CSLE ( $p=0.013$ for low $\mathrm{C} 3$ and $p=0.0004$ for low $\mathrm{C} 4$ ). Among the several different pharmacological tools, hydroxychloroquine (HCQ, $p=0.021,95 \%$ confidence interval (Cl) 0.0084-0.1007), azathioprine (AZA, $p=0.0217,95 \% \mathrm{Cl} 0.013-$ 0.1703 ) and cyclophosphamide (CTX, $p=0.0305,95 \% \mathrm{Cl} 0.013-0.170$ ) were identified as protective.

At follow-up, patients most commonly developed new haematological and cutaneous involvements, which were diagnosed in 43 and 11 patients, respectively. A trend towards statistical significance emerged for low $\mathrm{C} 4 \mathrm{lev}-$ els to predict new haematological involvement at follow-up ( $p=0.064$, chisquared: 3.42). Differently, positivity for antibodies against dsDNA emerged as the only predictor of the onset of cutaneous manifestations during follow-up ( $p=0.022$, chi-squared: 7.62). Low C3 levels approached statistical significance in the prediction of skin involvement $(p=0.058$, chi-squared 5.68).

Conclusion: According to the data from our monocentric cohort of 100 patients, complement and anti-dsDNA antibodies are the most accurate tools to predict disease progression in CSLE. HCQ, AZA and CTX reduce the rate of disease progression.

Table 1. Clinical manifestations, laboratory features and treatment details of recruited patients at diagnosis.

Number of patients

Skin manifestations

Skin manifestations
Haematological involvement

Lupus nephritis

Musculoskeletal involvement

Serositis

Neuropsychiatric involvement

Anti-nuclear antibodies

Anti-Ro antibodies

Anti-Sm antibodies

Anti-dsDNA antibodies

Anti-phospholipid antibodies

Hydroxychloroquine

Azathioprine

Cyclophosphosphamide

Micophenolate mofetil

Cyclosporine

Rituximab

Belimumab

Steroids

44

51

16
72

72
14

14

90

90
11

11
13

67
29

29

40

40
29

13
15

15

Disclosure of Interests: None declared

DOI: 10.1136/annrheumdis-2021-eular.2051

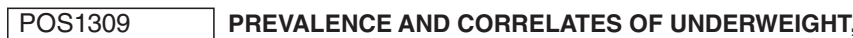 OVERWEIGHT AND OBESITY AMONG PATIENTS WITH JUVENILE IDIOPATHIC ARTHRITIS (JIA): EVIDENCE FROM THE NATIONAL PAEDIATRIC RHEUMATOLOGIC DATABASE (NPRD)}

F. Milatz ${ }^{1}$, J. Klotsche ${ }^{1}$, M. Niewerth ${ }^{1}$, J. Hörstermann ${ }^{1}$, D. Windschall ${ }^{2}$, F. Weller-Heinemann ${ }^{3}$, F. Dressler ${ }^{4}$, R. Berendes ${ }^{5}$, J. P. Haas ${ }^{6}$, G. Horneff ${ }^{7,8}$, K. Minden ${ }^{1,9} .{ }^{1}$ German Rheumatism Research Centre, Epidemiology and Health Care Research, Berlin, Germany; ${ }^{2}$ Northwest German Center for Rheumatology, Clinic of Paediatric and Adolescent Rheumatology, St.-JosefStift, Sendenhorst, Germany; ${ }^{3}$ Klinikum Bremen-Mitte, Prof.-Hess-Kinderklinik, Bremen, Germany; ${ }^{4}$ Children's Hospital, Medical School Hannover, Department of Paediatric Pneumology, Allergology and Neonatology, Hannover, Germany; ${ }^{5}$ Kinderkrankenhaus St. Marien, Paediatric Rheumatology, Landshut, Germany; ${ }^{6}$ German Center for Paediatric and Adolescent Rheumatology, Centre for Pain Therapy of Young People, Garmisch-Partenkirchen, Germany; ${ }^{7}$ Sankt Augustin Asklepios Children's Hospital, Division of Paediatric Rheumatology, Sankt Augustin, Germany; ${ }^{8}$ University Hospital of Cologne, Department of Paediatric and Adolescent Medicine, Cologne, Germany; ${ }^{9}$ Charité University 